\title{
METAMORFOSI DELL'ANIMA. CORPO, VITA E COSCIENZA
}

\author{
LUCA VANZAGO (*)
}

SunTO. - La riflessione sulla nozione di coscienza che Faustino Savoldi ha portato avanti per molti anni, e il cui frutto maggiore è l'opera La coscienza pubblicato nel 2013, era proseguita dopo tale pubblicazione. In conversazioni personali con lui, si era insieme giunti all'idea che il problema della coscienza e la questione del rapporto tra mente e corpo dovessero essere affrontati alla luce di una meditazione sul concetto di vita. Purtroppo non c'è stato il tempo per concludere tale lavoro. Questo saggio tenta di delineare alcuni presupposti teorici per proseguire comunque lungo la direzione intravista da Savoldi, e per portarne avanti così l'eredità intellettuale.

$$
* * *
$$

ABSTRACT. - The reflection on the notion of consciousness that Faustino Savoldi has carried out for many years, and whose greatest result is the work La coscienza, published in 2013, had continued after this publication. In personal conversations with him, we had come to the idea that the problem of consciousness and the question of the relationship between mind and body should be addressed in the light of a meditation on the concept of life. Unfortunately there was no time to complete this work. This essay tries to outline some theoretical assumptions to continue in any case along the direction glimpsed by Savoldi, and to bring forward his intellectual legacy.

Nel corso delle conversazioni che periodicamente si tenevano a casa sua per discutere delle questioni connesse alla pubblicazione del suo lavoro sulla coscienza, Faustino Savoldi e io ci siamo imbattuti, con frequenza crescente, nel problema della vita. L'opera cui stava attendendo da anni gli aveva consentito di discutere ravvicinatamente le opere dei maggiori filosofi della mente anglosassoni, come Searle e

${ }^{(*)}$ Dipartimento di Studi Umanistici, Sezione di Filosofia, Università degli Studi, Pavia, Italia. E-mail: luca.vanzago@unipv.it 
Dennett per non citare che due nomi famosi, oltre a una mole davvero impressionante di altri scritti tra cui, come è noto, spiccano i lavori della scuola fenomenologica cui Savoldi aveva già dedicato un libro che meriterebbe di essere ripubblicato, il cui titolo volutamente reticente suona "introduzione alla psichiatria fenomenologica". Nel suo costante sforzo di ripensare il rapporto tra mente e cervello, Savoldi aveva posto con chiarezza molte questioni relative allo statuto della coscienza, tra cui la necessità di distinguere coscienza fenomenica e autocoscienza, da una parte, dalla veglia e dagli stati cerebrali dall'altra. Ma restava costantemente aperto il problema di come unire ciò che era stato separato, nella convinzione che ciò dovesse essere fatto con un'ottica diversa da quella riduzionistica ma al contempo evitando le aporie del dualismo.

Il tema della vita, mi disse, lo faceva tornare con la mente ai suoi giovanili studi crociani, all'ultimo Croce in particolare, e alla sua ripresa di questa problematica nel contesto della discussione critica ma mai polemica con Enzo Paci e la sua interpretazione della filosofia di Vico. Nel suo libro Ingens Sylva, Paci intende mostrare la presenza in Vico di un pensiero della natura, che tolga all'interpretazione prevalente, ossia sostanzialmente quella crociana e storicistica, la forza di assolutezza che in quell'epoca tale interpretazione possedeva. Conformemente agli interessi teoretici di Paci, il problema si palesava come esigenza di intendere il radicamento naturale della soggettività umana, anche storica, e correlativamente di pensare la natura stessa come dotata di una propria storicità. Sono temi che emergeranno più tardi, negli anni Cinquanta, nel contesto della proposta teorica paciana nota come relazionismo, in cui gioca un ruolo fondamentale la nozione di tempo come processo irreversibile. Savoldi fu testimone diretto di tale evoluzione del pensiero di Paci, di cui fu amico ma mai mero ripetitore, come ebbe a dirmi raccontandomi anche qualche gustoso aneddoto personale.

Ma la questione della vita rimane al di sotto degli eventi storici e personali come vero e proprio residuo impensato della filosofia della coscienza e della mente, il che è vero, si può ora dire, sia per la versione anglosassone di stampo analitico sia anche per la versione continentale di marca fenomenologica, a cui come è noto Savoldi è molto più vicino in tutto l'arco della sua riflessione. In questo senso, il compimento del grande libro sulla coscienza non poteva che rappresentare un nuovo inizio: verso una riflessione centrata appunto sul concetto di vita come quello capace di tenere insieme corpo e coscienza.

Non c'è stato purtroppo modo di andare molto in là in questo 
progetto, che tuttavia aveva suscitato in Savoldi una forte simpatia. Ora mi pare che in un certo senso questo tragitto speculativo disegni una parabola significativa e non casuale se si considera come il pensiero occidentale abbia trattato il tema dell'anima, e quindi dell'esperienza cosciente, a partire almeno da Platone, che Savoldi colloca nel suo libro sulla Coscienza all'origine della consapevolezza filosofica della irriducibilità dell'esperienza alla corporeità materiale. Mi è parso utile dunque ripercorrere rapidamente alcuni tratti salienti e certi snodi significativi della meditazione filosofica sull'anima per vedere come in un certo senso essa disegni un cerchio che si chiude con la riscoperta della vita come esperienza corporea, e quindi indichi una direzione di ricerca che consuona con le riflessioni poco fa evocate.

Si è già detto di Platone, e in particolare della sua strategia, attuata nel Fedone, volta a dimostrare l'immortalità dell'anima come vita essenziale, che non può dunque morire, anche quando il corpo scompare. Ma l'idea di qualcosa che possa essere "animato" non nasce con la filosofia. L'antropologia ha coniato un termine, sia pure oggi messo in discussione, per descrivere l'atteggiamento dei popoli cosiddetti "primitivi" nei confronti della realtà: animismo. In ciò che può ancora avere di valido, tale termine vale a esprimere la convinzione secondo cui la realtà non è inerte, ma essa stessa, e tutto ciò che in essa esiste, vive e agisce. I fiumi, gli alberi, i luoghi e perfino gli oggetti sono visti come dotati di un principio di animazione. Per non parlare poi, naturalmente, degli animali, di quegli esseri cioè che mostrano evidentemente di possedere qualche forma di movimento interno, che conduce e consente poi i movimenti esterni. Ma come spiegare, come farsi una ragione, di tale attribuzione di un'anima alla realtà? E si può parlare nello stesso modo dell'anima dei luoghi, di quella di un fiume, dell'animazione propria del regno vegetale o di quello animale? L'animismo, è stato detto, consiste nella proiezione ingenua di caratteristiche umane su enti inanimati. Ma un albero non è inanimato, e lo zampillare di una fonte dà l'i $\mathrm{i}$ dea di un evento, di una trasformazione, cioè del contrario dell'inerzia.

Molti reperti archeologici testimoniano poi della convinzione che anche gli spazi in cui gli uomini si trovavano a vivere possedessero per loro delle caratteristiche non "neutre", come l'uomo moderno tende a pensare, ma peculiari e dinamiche. La fondazione di un nuovo nucleo urbano veniva condotta secondo complessi rituali tendenti a determinare le forze presenti nel luogo previsto. La connessione tra gli esseri umani e le forze "naturali", o anche a volte sovrannaturali, era vissuta 
direttamente e non costituiva una interpretazione successiva. Tuttavia tale connessione non si dà più, almeno in Occidente e almeno se si considera l'esperienza comune; e tale scomparsa di un atteggiamento testimoniato da tutte le culture antiche costituisce in sé un problema da spiegare, senza propendere per una intrinseca ingenuità da "primitivi" che avrebbe attardato tali sistemi culturali, ma senza neppure sognare una improbabile riscoperta di verità sepolte da millenni e superiori alle miserie attuali.

Un elemento da considerare attentamente è rappresentato dal nesso, nelle culture antiche, tra concezioni dell'anima e convinzioni relative ai sogni, alle premonizioni, alle fantasie e fantasticherie (che spesso naturalmente non erano intese in questo senso "moderno", ma come vere visioni). C'è un dato molto interessante che emerge dall'attuale ricerca nel campo dell'antropologia delle religioni, per cui probabilmente ogni popolo in ogni tempo ha avuto una qualche forma di attività "psicotropa" (termine quanto mai anacronistico se usato per le popolazioni antiche, come si vedrà), ossia una qualche forma organizzata di esperienza esoterica, tendente a superare o in ogni caso a modificare l'esperienza normale e quotidiana. Molte religioni misteriche sono basate su tali pratiche, che dovevano consentire agli iniziati di superare i limiti "apparenti" della propria condizione, per poter scoprire la verità sulla propria natura. In tal senso, i legami sensoriali tra gli uomini e il mondo circostante venivano vissuti come limitanti e opprimenti, fonte più di errore che di verità.

Questa concezione dell'esperienza, implicitamente dualistica, aveva una relazione diretta e profonda con il problema della morte. L'essere umano è tale in quanto sa di essere mortale. Il senso di questo evento misterioso, la possibilità che esso non significhi la fine di tutto, il destino eventuale di ciò che può superare tale soglia, è un problema che ha originato (e continua a produrre) un notevole ventaglio di speculazioni e di teorie sulla condizione umana, e sul rapporto tra la natura corporea dell'essere umano e qualcosa che può non dipenderne totalmente. In questo senso gioca un ruolo esemplare il culto dei morti e l'universalità dei riti di sepoltura. Le esperienze naturali e quelle sovrannaturali non hanno, a questo punto della storia della cultura umana, ancora un limite chiaramente determinato, anche se questo non hai mai significato confondere i due piani. La vita terrena viene vista come quella vera, che sarà rimpianta dopo la morte; e però la vita dopo la morte è considerata come un mistero ma non come una mera illusione. Questa 
ambivalenza segna molte culture antiche, e in particolare quelle che, come si vedrà nel prossimo paragrafo, sono connesse alle origini della riflessione filosofica: quella greca arcaica e quella ebraica.

Questi interrogativi sul destino dell'esistenza, sulla sua condizione e sulle sue caratteristiche, costituiscono già implicitamente delle riflessioni che segnano un distacco dalla pura naturalità. L'essere umano si interroga sul mistero della propria morte, sulle origini della propria esistenza e sugli eventi che ne segnano il corso, e così facendo inizia una poi sempre più articolata riflessione sulla natura e sulla definizione delle stesse nozioni di origine, causa, e destino. L'articolazione di questa riflessione, il passaggio cioè dal tentativo di dare una risposta a domande pressanti a quello di dar conto di come elaborare le risposte stesse, segna la soglia superata la quale si entra nel mondo filosofico. Il problema si sposta, e si approfondisce. La domanda sulla natura umana diventa anche una domanda sull'idea di natura umana, e poi sull'idea di idea. Questo non implica tagliare i ponti con quanto sia venuto "prima"; le influenze sono anzi molto importanti e a volte sorprendenti. Ma la riflessione filosofica sull'anima nasce quando qualcuno comincia a chiedersi "a quali condizioni" si possano produrre delle risposte alle domande che lo assillano. E questa è la prima, ma probabilmente cruciale, delle varie trasformazioni a cui la nozione di anima è stata soggetta.

Nella sua sintesi e revisione della dottrina di Socrate, Platone si confronta anche coi suoi contemporanei, e in particolare con Democrito, che rappresenta una potente prospettiva filosofica di stampo materialistico, capace di generalizzare la dottrina eleatica in modo creativo e originale attraverso la nozione di atomo e una visione fortemente deterministica della realtà. In Democrito confluiscono gli esiti della filosofia naturalistica degli Ionici e gli aspetti più "cosmologici" dei primi pluralisti, Empedocle ed Anassagora, e al contempo si realizza una sintesi radicale della concezione ciclica e fatalistica del mondo propria della mentalità ellenica, che viene espressa da questo pensiero in una forma particolarmente distillata e pura, la quale lascia coerentemente fuori dal proprio quadro categoriale qualsiasi appiglio all'idea di fine o telos.

Platone reagisce dunque con forza a tale prospettiva, riaffermando al contrario, con una forza però incomparabile, l'ipotesi finalistica, rielaborando a suo modo la grande riflessione sofistica sulla soggettività, e sganciando il logos da qualsiasi dipendenza dalla physis. In particolare, la strategia che consente a Platone di raggiungere risultati inusitati si fonda sulla comprensione della capacità del logos di dar ragione 
di sé, di auto-giustificare le proprie determinazioni, cioè di "fondare" la propria ricerca della verità e con ciò toglierla al relativismo protagoreo, $\mathrm{ma}$ anche contemporaneamente alla cieca necessità atomistica.

In questo quadro Platone opera poi un'ulteriore trasformazione, integrando l'affermazione socratica dell'universalità del pensiero come essenza dell'anima entro una prospettiva insieme epistemologica e ontologica, in cui l'anima trova una definizione univoca come principio d'ordine, di moto e di finalizzazione cosciente e interiore. Egli può così esplicitare tutte le conseguenze che derivano dall'aver concepito l'anima come luogo di manifestazione dell'idealità, della verità che non muta di contro alla mutevolezza delle opinioni. In tal modo egli preserva (e quindi combatte sul suo stesso terreno) la determinazione eleatica e poi atomistica dell'essere come ciò che è immutabile ed eterno, trasferendo però tali determinazioni sul piano dell'idealità di contro alla realtà empirica intesa come mutamento sia ontologico che gnoseologico, secondo l'equazione espressa dalla metafora della linea nella grande meditazione condotta nella Repubblica.

Aristotele svolge un ruolo storico generale di sistematore e al contempo revisore critico delle dottrine platoniche che si conferma anche nel caso della concezione dell'anima. Nei suoi lavori specificamente dedicati a tale questione, e in particolare nel Perì Psychés (De Anima), egli ad un tempo rifonda e codifica tutti gli aspetti essenziali della "psicologia" platonica in un quadro culturale e speculativo diverso, che fa loro assumere un senso nuovo. Aristotele conferma la posizione platonica nell'assegnare un fondamento ontologico all'antropologia, nel saldarlo con le tesi principali della metafisica, nell'attribuire una funzione cosmica essenziale all'anima basata sulla sua attività conoscitiva, nel trarre conclusioni di natura finalistica da questa determinazione conoscitiva. Anche l'aspetto di auto-interpretazione critica dell'essere dell'uomo attraverso l'attività dialettica della parte razionale dell'anima viene ribadito e anzi semmai rafforzato dallo sganciare tale attività dalla contemplazione delle idee per affidarlo ad una concezione più costruttiva (poietica) del logos.

La scienza dell'anima che deriva da questa impostazione si basa sulla considerazione astrattiva dei dati empirici, ma fondata sulla capacità intellettuale di trascenderli nella loro limitatezza, secondo l'idea già platonica dell'universalità del logos come possibilità di auto-fondazione. In Aristotele tale posizione consente allora di attenuare il contrasto netto, istituito da Platone, tra anima come principio di vita e moto e 
anima come principio e sede del pensare, perché è quest'ultimo ad essere visto come processo attivo che però ha inizio e comunque rapporto, nell'uomo, con la sfera sensibile e vitale stessa. Questa trasformazione passa per una complessa dottrina del moto, delle cause, della struttura potenza-atto-privazione. Ciò che infatti muta in Aristotele, rispetto a Platone, è la natura del rapporto ontologico, e quindi funzionale ed etico, dell'anima nei confronti della corporeità e conseguentemente della sfera mondana.

L'anima non è più vista come "demone" di natura ideale e non materiale, aspirante a ritornare a tale condizione di libertà e trascendenza. L'anima viene ora definita, secondo le coordinate metafisiche aristoteliche, come atto primo di un corpo organico avente la vita in potenza. Il suo status ontologico la fa rientrare all'interno della legge generale dell'ilemorfismo aristotelico (cioè della concezione della realtà in quanto basata su sostanze che sono un composto di materia e di forma), e nella teoria del divenire come realizzarsi dell'essere in atto già potenzialmente presente nella natura. In questo divenire l'anima è - in ogni vivente - insieme principio motore e causativo, e scopo e perfezione finale (entelechia). Il suo essere forma di un corpo che ha la vita in potenza, inoltre, la configura non come sostanza separata (come in Platone almeno nella sua tendenza più dualistica) ma come intrinsecamente associata alla corporeità. Il primato della causa finale riafferma il valore dell'anima in generale nel mondo vivente e in particolare nell'uomo, permettendo di fondare l'eticità sugli stessi principi dell'ontologia e dell'antropologia. Inoltre conferma anche il dominio del pensiero, la sua capacità causativa entro la realtà naturale.

L'anima in Aristotele è tripartita come in Platone, ma secondo una revisione fondamentale in senso ilozoistico della posizione platonica: non si tratta di opporre le parti ma di comprenderne le reciproche interazioni e soprattutto la natura esatta della gerarchia. Aristotele distingue un'anima vegetativa (deputata al nutrimento e alla riproduzione), un'anima sensibile, propria anche degli animali, che svolge attività di percezione e immaginazione, ed un'anima razionale, propria soltanto dell'uomo. Il modello è perciò quello di una scala ascendente delle funzioni e prerogative psichiche, a cui si accompagna una riduzione del numero di individui che ne sono caratterizzati. Tutti i viventi come tali sono caratterizzati dall'anima "inferiore"; solo alcuni, gli animali, anche di quella intermedia; e soltanto pochi, gli esseri umani, anche di quella superiore. 
La concezione aristotelica dell'anima è prevalentemente funzionalistica, cioè è intesa in termini di capacità del corpo di agire, di percepire, ed eventualmente di pensare. L'anima consente inoltre l'unificazione delle funzioni corporee. Da questo punto di vista la prospettiva aristotelica è essenzialmente biologica. Il grande filosofo tenta in effetti una sintesi ardita tra concezioni diverse dell'anima, superando gli opposti riduzionismi che vogliono l'anima a volte come espressione di funzioni esclusivamente vitali (secondo le concezioni più arcaiche), altre volte come presenza del divino nell'uomo, come nel caso dei dualismi "orfici". Per realizzare tale sintesi Aristotele mette in campo una raffinata dottrina "strutturale" dell'anima, vista come essenza (ousia) del corpo vivente, sua "causa" (aitia), principio (archè) e fine (telos). In questo egli applica in modo coerente e particolarmente brillante la propria concezione metafisica generale relativa alla realtà e all'essere. Rimane però così aperta la questione dello statuto ontologico di qualcosa che, essendo "soggetto" in senso moderno (cioè non in senso aristotelico), e non soltanto oggetto, sembra richiedere una comprensione ontologica che è appunto lasciata irrisolta entro le categorie aristoteliche. In Platone come in Aristotele, come in generale nella filosofia greca fino a Plotino, l'anima è sì individuata nella sua specificità ontologica, ma manca una tematizzazione esplicita sull'essere dell'anima in quanto incomparabile rispetto a quello degli altri enti. Chiara in questo senso è la posizione di Aristotele, per cui la struttura ontologica formamateria e potenza-atto viene fatta valere senza residui anche nel caso dell'anima, senza che venga mai posto il problema se essa sia con ciò effettivamente preservata nella propria condizione ontologica peculiare, oppure concepita pur sempre alla stregua di un ente, sia pure sui generis. Che l'anima, o io, o soggettività, non sia un ente, è ciò che la metafisica aristotelica non consente in definitiva di pensare.

Con il diffondersi del messaggio cristiano il quadro cambia in modo sostanziale. L'anima non può più essere vista semplicemente come principio vitale di "questa" vita, in quanto essa vale soprattutto a garantire quella successiva, quella vera. Di qui ad un tempo l'affinità e la differenza con la concezione platonica e con essa tutta la corrente "orfica" antica. È tale differenza a fare anche del rinnovamento, implicato dall'adozione della fede cristiana, un passo diverso da quello della partecipazione a un rito misterico come quello di Eleusi. Senza sminuire le somiglianze strutturali, che caratterizzano del resto tutte le pratiche religiose di rinnovamento dell'antichità, si può notare però che il 
messaggio cristiano dà al processo di trasformazione (che è segnato dal battesimo) un senso nuovo. La consapevolezza del senso dell'esistenza che si vuole ottenere nella prospettiva cristiana è ad un tempo di una destinazione ulteriore che si acquisisce però "qui e ora", conferendo così anche alla vita terrena un senso differente, una "direzione" e una teleologia inedite, nonché possibili a tutti, cioè universali.

In questo senso è la stessa idea della morte che cambia di segno: l'antropologia cristiana fa della morte un passaggio che riguarda tutti (quindi non solo gli iniziati) e proprio perciò è universalizzabile e non riguarda soltanto chi "sa", ma semmai chi crede e si converte alla vera fede. Essendo un dono del Dio creatore, la vita ultraterrena non dipende che dal Suo giudizio, non quindi da quello umano. Rispetto alla concezione classica greca dell'anima emergono pertanto alcuni caratteri distintivi della antropologia cristiana: la sua realtà e individualità personale originaria, derivante dall'atto stesso diretto della creazione: essa deriva dall'atto di Dio, e non è generata per divisione, disseminazione, partecipazione a una anima del mondo; la sua distinzione ma insieme non opposizione al corpo, che è anch'esso originariamente creato da Dio, e quindi non ha in sé un valore negativo e deteriore, ma è destinato a essere dominato e sviluppato dall'anima; il carattere secondario e storicamente derivato di una relativa contrarietà corpo-anima (passioni, dolore, morte), che deriva da una scelta etica negativa (il peccato) non necessaria né permanente, ma annullabile grazie alla condotta proba e all'aiuto divino (il che in qualche modo dà un senso al dolore); la storicità concreta e personale del destino dell'anima, individualmente distinto e concepito in senso non necessitante, non coinvolto nel ciclo del tutto cosmico, ma dipendente ad un tempo dalla provvidenza divina e dalla scelta umana: è questa forse la svolta più radicale del "pensiero" cristiano, che sposta il centro della prospettiva dal cosmo all'uomo nella sua diretta relazione al divino, e quindi dall'ontologia all'etica. In questo senso il messaggio cristiano riprende il pensiero ebraico. Rispetto all'ebraismo e al primato del tempo futuro (il messia che deve ancora venire), tuttavia, il cristianesimo pone il primato del presente come conversione alla vera vita sulla base dell'evento della morte e resurrezione di Cristo. È questo che produce la differenza con l'ebraismo e la nascita di un pensiero della storia come fondazione della città umana a immagine di quella celeste, la cui realtà è garantita dalla resurrezione.

Con la rivoluzione scientifica moderna il quadro cambia radicalmente. Il pensiero moderno opera una decisa scelta nella fondazione di 
una nuova antropologia filosofica, in seguito alle trasformazioni imposte dalla rivoluzione scientifica. Tale fondazione non è tuttavia innanzi tutto possibile in quanto relativa a una scienza autonoma, ma coinvolge in maniera globale la problematica del metodo e della fondazione del sapere in generale. La centralità cosmica dell'anima, affermata nel Rinascimento, viene così ribadita e anzi emerge progressivamente l'importanza di quel particolare universo storico-culturale che è il sapere umano come tale. Ai suoi inizi, in particolare, il pensiero moderno segue i binari delineati dalla filosofia rinascimentale, che pongono due tendenze tra loro opposte: da una parte la prospettiva naturalistica, dall'altra quella spiritualistica. Tale divisione è però trasformata in forza della nuova coscienza critica propria della prospettiva derivante dalla nascita del metodo scientifico, in una duplice e alternativa concezione dell'oggettività e della verità, che coinvolge interamente la questione dell'interpretazione dell'anima.

Per un verso infatti la realtà vera corrisponde all'oggetto acquisito ed empiricamente verificato coi metodi di misurazione e di controllo matematico propri della nuova fisica. Sicché esso è ricondotto alle sue qualità "primarie" che competono alla materia in movimento e come tali costituiscono il modulo della certezza del reale e della sua conoscibilità scientifica, secondo la prospettiva inaugurata da Galileo. L'anima da questo punto di vista è allora, rispetto ai fenomeni fisici, un elemento perturbante, in quanto introduce apparenze soggettive. Inoltre costituisce un elemento limitante, in quanto è ammessa come realtà indubitabile, ma inconoscibile scientificamente e quindi da abbandonare alla religione e alle regole pratiche della vita. Tale scissione non può peraltro rimanere a lungo tale, in quanto con l'Illuminismo si avvia la ricerca di una applicazione della metodologia scientifica anche alla sfera psichica ed etico-storica.

D'altro canto, in quanto oggetto pensato come primo e puro, e quindi ricondotta alla determinazione del pensiero e spogliata di ogni sensibilità e organicità, ossia come "res cogitans", l'anima diventa modello e fondamento dello stesso schema interpretativo dell'interpretazione matematica e meccanica della natura. L'anima viene resa il luogo della verità assolutamente indipendente da ogni premessa e presupposto, e secondo il metodo della chiarezza e distinzione coincide con lo stesso atto di pensiero. Elevata a questa funzione, tuttavia, l'anima perde la sua concretezza e integrità. Non essendo più essenzialmente forza animatrice del sensibile, della corporeità, non essendo più, 
tomisticamente, un principio organico di finalizzazione dall'interno e spontaneamente della realtà materiale, perde anche la propria caratteristica di essere principio volontaristico di libertà, scelta e decisione. Diventa invece il principio e la sede di manifestazione della pura necessità razionale, dell'evidenza conoscitiva. Agisce pertanto direttamente soltanto su se stessa mentre per essere efficace sulla materia necessita di una mediazione.

Si inaugura così nel pensiero moderno una scissione, che spesso si moltiplica in una stratificazione di opposizioni compresenti. Come soggettività autocosciente l'anima viene intesa, a livello metodologico critico, come principio di fenomenizzazione, cioè fondamento e criterio di certezza di ciò che si manifesta. Come principio di conoscenza essa è sede di evidenza necessaria e in questo senso non può più affermarsi come principio pratico di spontaneità e libertà se non attraverso una rifondazione ontologica (che avviene in modo coerente con Spinoza). La sua realtà ontologica è determinata essenzialmente come quell'azione del tutto peculiare che è il pensare (cogito, res cogitans). Può allora sì conoscere la realtà esterna (res extensa) ma soltanto come idea, non immediatamente, quindi non nella sua reale alterità, se non tramite il farsi presente ad essa dell'Assoluto come garanzia di corrispondenza tra idea e "cosa esterna". Sempre grazie al tramite dell'Assoluto può allora agire sulla sfera corporea, ma si tratta perciò di una azione non "vera", perché il dualismo ontologico conduce a impedire l'azione diretta dell'anima sul corpo. Ne deriva in questo senso il tentativo di tutto il razionalismo di pensare il parallelismo mente-corpo (si pensi a Leibniz ma anche all'occasionalismo). Si pone allora il problema della crisi di pensabilità dell'unità e individualità dell'uomo, nonché quello della pensabilità della comunicazione tra sostanze pensanti, cioè il problema dell'intersoggettività.

Naturalmente la figura emblematica di questa configurazione teorica è Cartesio. La dottrina dell'anima per Cartesio non appartiene alle scienze della natura, ma è scienza di una realtà extransensibile. La natura dell'anima fonda la possibilità di ogni scienza, e perciò permette all'uomo di spingere lo sguardo oltre i limiti della scienza stessa, verso le problematiche metafisiche. Nell'indagine metafisica sulla sostanza spirituale e intelligibile la ragione dà il contributo del suo rigore dimostrativo alle verità della fede.

L'impostazione cartesiana pone però con ciò anche il problema del dualismo di anima o spirito e materia, dualismo declinato in manie- 
ra "pura" alla luce della nuova teoria della conoscenza. La connessione immediata tra l'autoconsapevolezza propria del pensiero e la sua considerazione in quanto sostanza incorporea traspare sin dal Discorso sul metodo, laddove, nella quarta parte, esaminando le ragioni che provano l'esistenza di Dio e dell'anima umana, Cartesio sostiene di poter fingere di non avere un corpo, ma di non poter fingere di non esistere affatto. L'esistenza viene così connessa alla determinazione della sua certezza, e questa a sua volta alle procedure intrinseche dell'anima.

Di contro a tale determinazione, emerge la comprensione del corpo come materia sottoposta alle leggi fisiche. Questo si applica anche al corpo umano. Lo statuto della corporeità è quello dell'estensione e quindi dell'esteriorità reciproca delle parti. È questa la ragione concettuale che porta alla determinazione del corpo "proprio" (il corpo di colui che dice io) in termini assolutamente analoghi a quelli di tutti gli altri corpi.

Riemerge pertanto il dualismo platonico, sotto una forma, tuttavia, del tutto inedita: è la determinazione dell'auto-conoscibilità a operare la scissione e lo spartiacque tra res cogitans (che è cogitans perché pensa se stessa) e res extensa. Il corpo non essendo "animato", i suoi movimenti sono spiegabili in modo necessario e sufficiente attraverso l'immagine della macchina. Il corpo umano è assimilato a quelli animali $\mathrm{e}$ in generale alla materia come ciò che è sottoposto a leggi di natura esclusivamente meccanicistica.

La storia delle riflessioni successive non può essere neppure minimamente esaminata qui, se non per dire che con Cartesio si configura in modo sostanzialmente definitivo, giungendo fino a noi, il problema della comprensione del nesso tra anima e corpo. Ora è proprio tale nesso che resta impensato da Cartesio in avanti, e che soltanto oggi, con una certa particolare versione delle neuroscienze chiamata emergentismo, sembra poter essere ricompresa in modo innovativo. Attraverso una revisione della nozione meccanicistica di corporeità, revisione ottenuta all'interno stesso delle ricerche neurobiologiche, è ora divenuto plausibile intendere la corporeità stessa come capace di esperienza, al contempo naturalizzando l'esperienza. Ma se questo è vero allora appare inevitabile rivedere l'impianto dualistico di ascendenza cartesiana anche nella sua versione per così dire mutilata, rappresentata dal riduzionismo psico-fisico, che di quel dualismo non tiene per vero che uno dei due lati, limitandosi a sopprimere l'altro ma poi ereditando così il problema di come spiegare che la mera materia possa anche pensare. 
In autori come Francisco Varela (inventore dell'espressione "neuro-fenomenologia") si fa invece largo l'ipotesi che sia proprio la struttura categoriale dualistica che resta come un residuo anche nell'approccio riduzionistico attuale a costituire un ostacolo epistemologico, come avrebbe detto Bachelard, che impedisce di comprendere effettivamente ciò che si sta progressivamente scoprendo. L'ultima metamorfosi dell'anima sembra allora essere costituita da un ritorno al corpo vivente, dove il participio presente segnala proprio l'esigenza per il pensiero di comprendere la vita nel suo accadere, e non come cosa morta pronta a essere dissezionata, secondo l'immagine del tavolo anatomico magistralmente simboleggiata dai quadri di Rembrandt. Mi pare che sia in questo contesto che le ricerche neurobiologiche e la riflessione filosofica possano tornare effettivamente a parlarsi, in un quadro di rinnovamento concettuale che non fa che rilanciare la sfida a pensare la vita, quale Faustino Savoldi aveva inteso dover costituire il compito futuro della ricerca, senza altri aggettivi. 